\title{
Optimization of Plant Regeneration in Different Pepper (Capsicum annuum L.) Lines
}

\author{
Tolga İzgü̈,a,*, Hülya İlbi ${ }^{1, b}$, Yeşim Yalçın Mendi ${ }^{2, c}$ \\ ${ }^{I}$ Department of Horticulture, Faculty of Agriculture, Ege University, 35040 Izmir, Turkey \\ ${ }^{2}$ Department of Horticulture, Faculty of Agriculture, Cukurova University, 01330 Adana, Turkey \\ *Corresponding author
}

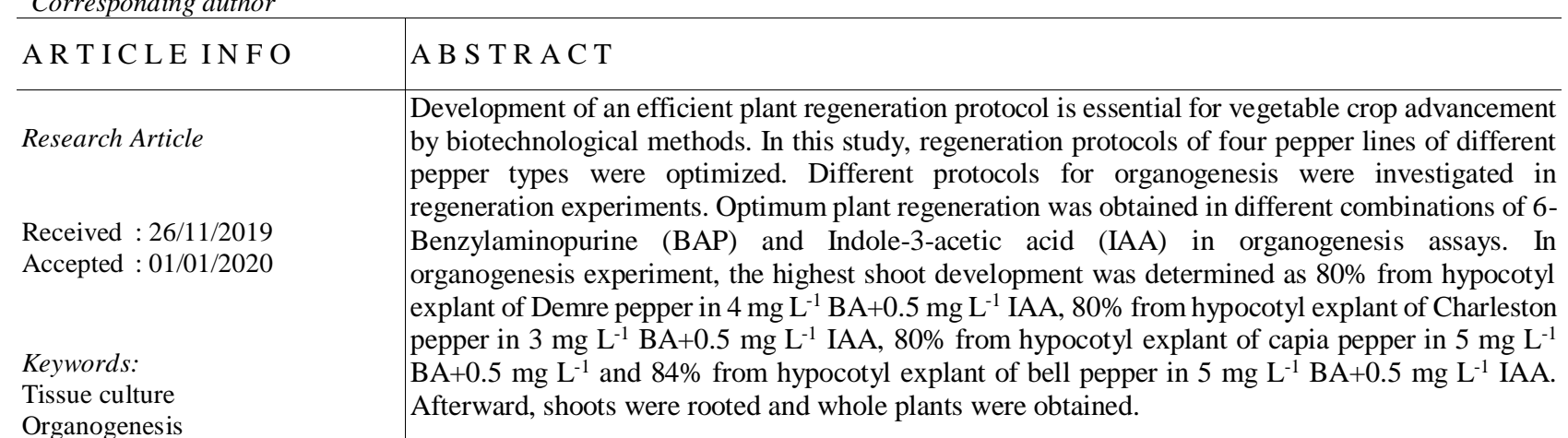

Plant growth regulators

Shoot formation

Callus

$\begin{array}{llll} & \text { iD http://orcid.org/0000-0003-3754-7694 } & \mathbf{b} \otimes \text { hulyailbi@gmail.com } & \text { (iD) https://orcid.org/0000-0002-7691-7996 } \\ \text { ymendi@gmail.com } & \text { (i) https://orcid.org/0000-0002-4587-5156 }\end{array}$

(c) (1) () This work is licensed under Creative Commons Attribution 4.0 International License

\section{Introduction}

Pepper (Capsicum annuum L.) is one of the most important and popular vegetables grown in Turkey and other countries around the world (Ochoa-Alejo and Ramírez-Malagón, 2001; Aboshama, 2011; El Nagar, 2012, Akyavuz et al., 2018). C. annuum L. is used as fresh or dried in different foods. Its nutritional properties, including antioxidants, are essential for human nutrition (Orlinska and Nowaczyk, 2015). Besides, pepper is also a source of natural colors and as medicine (Valadez-Bustos et al., 2009).

Capsicum seeds typically have short viability and might promptly loose germination capacity. Therefore, plant tissue culture techniques support a way for the clonal micropropagation of $C$. annuum L. (Sanatombi and Sharma, 2006; Orlinska and Nowaczyk, 2015). Besides, in vitro tissue culture followed by genetic transformation might be an simple, efficient and economic means for obtaining a large number of production, consistently uniform and true-to-type plants within a short span of time for improvement of yield and quality. There are many studies using different explants to improve the organogenesis protocol in the Capsicum genus. Different explants such as cotyledon and hypocotyl (Gunay and Rao, 1978; Agrawal et al., 1989; Arroyo and Revilla, 1991;
Hyde and Phillips, 1996; Christopher and Rajam, 1996; Franck-Duchenne et al., 1998; Husain et al., 1999; Venkataiah et al., 2003; Joshi and Kothari, 2007; Sanatombi and Sharma, 2008; Orlinska and Nowaczyk, 2015; Renfiyeni et al., 2017; Hegde et al., 2017a, b; Gammoudi et al., 2017; Bhutia et al., 2018), zygotic embryo (Agrawal and Chandra, 1983; Arous et al., 2001), young seedlings (Phillips and Hubstenberger, 1985; Ebida and $\mathrm{Hu}, 1993$ ), stem, leaf, root, shoot tip, embryo (Agrawal et al., 1989; Madhuri and Rajam, 1993; Christopher and Rajam, 1996; Venkataiah et al., 2003; Sanatombi and Sharma, 2007a, 2007b; Renfiyeni et al., 2017; Bhutia et al., 2018), mature seeds (Ezura et al., 1993), shoot tip (Madhuri and Rajam, 1993), injured hypocotyl (RamírezMalagón and Ochoa-Alejo, 1996), seedling and embryonal explants (Dabauza and Pena, 2001), epicotyl (Renfiyeni et al., 2017), shoot buds (Haque and Ghosh, 2018) were used for in vitro organogenesis of Capsicum.

However, it is noted that development of protocols is still needed because Capsicum genus is recalcitrant for both regeneration and genetic transformation (Liu et al., 1990; Steinitz et al., 1999; Kothari et al., 2010; Maligeppagol et al., 2016; Gammoudi et al., 2017). 
Several studies have been carried out to optimize regeneration in Capsicum genus. However, the most common problem is genotypic effect. The genotype has a strong effect in regeneration from various explants and this makes it necessary to optimize in vitro regeneration protocols for the specific genotypes and cultivars. Therefore, in this study, an attempt has been carried out to develop an efficient in vitro regeneration protocols for micro propagation of the popular lines of Capsicum types by using cotyledon and hypocotyl explants from in vitro regenerated plants.

\section{Material and Method}

\section{Plant Materials and Surface Sterilization}

Seeds of four C. annuum L. pure lines, 'Green pepperF614', 'Charleston pepper-F22', 'Capia pepper-M801' and 'Bell pepper-F46' were obtained from AD-Rossen Africulture Company (Antalya, Turkey). Seeds were washed under tap water for 20 min, dipped in $70 \%$ ethanol for $1 \mathrm{~min}$, washed three times with sterile distilled water and then immersed in $20 \%$ sodium hypochlorite solution (Domestos ${ }^{\circledR}$ commercial bleach solution with $4.5 \%$ active chlorine, v/v, $\mathrm{NaOCl}$ ) with 1-2 drops of Tween-20 for 20 min. Finally, seeds were rinsed three times with sterile distilled water. Sterilized seeds were then placed in Plant Growth Regulators (PGRs) free-MS basal medium containing $30 \mathrm{~g} \mathrm{~L}^{-1}$ sucrose in petri dishes for germination. Ten seeds were placed in each petri dish. The petri dishes were then incubated in a growth chamber condition till seeds produce young seedling.

\section{Explant Preparation and Shoot Induction}

After eleven days, the germinated seeds produced young seedlings having cotyledon leaves. Hypocotyls (2-3 $\mathrm{mm}$ in length, Figure 1A) and cotyledons (1-2 mm length, Figure 1B) from 11-day-old seedlings were excised into pieces using sterilized surgical blades. Four segments were placed horizontally in each petri dishes and gently pressed into MS basal medium (Murashige and Skoog, 1962) supplemented with $30 \mathrm{~g} \mathrm{~L} \mathrm{~L}^{-1}$ sucrose, $1 \mathrm{mg} \mathrm{L}^{-1}$ 2-(N-morpholino) ethanesulfonic acid (MES), $3.5 \mathrm{~g} \mathrm{~L}^{-1}$ gelrite and various combinations of PGRs; BAP $\left(1,2,3,4\right.$ and $\left.5 \mathrm{mg} \mathrm{L}^{-1}\right)$ and IAA $\left(0.5,1 \mathrm{mg} \mathrm{L}^{-1}\right)$. Shoots (1-2 cm length) were obtained from the explants and placed vertically in the MS basal media supplemented with $0.5 \mathrm{mg} \mathrm{L}^{-1}$ Gibberellic acid $\left(\mathrm{GA}_{3}\right)$ for elongation of shoots. The culture tubes containing shoots were placed under fluorescent light in a growth chamber $\left(24 \pm 2^{\circ} \mathrm{C}\right)$ under $16 \mathrm{~h}$ photoperiod. After shoot initiation and elongation, they were subcultured again on freshly sterilized medium containing $0.5 \mathrm{mg} \mathrm{L}^{-1}$ IAA for root initiation.

\section{Experimental Design and Statistical Analysis}

All experiments were set up in a completely randomized factorial design and repeated twice with four replicates per treatment. Callus and shoot percentage was calculated as the number of explants on which callus or shoot formed/total number of explants. All quantitative data expressed as percentages were subjected to arcsine transformation. Means were separated by analysis of variance and the least significant difference (LSD) test was performed to examine differences in callus formation and shoot production among different explants in each
Capsicum lines. All data analysis was examined using the JMP program (SAS Institute, Cary, NC) ver. 5.00 and significance was considered at $\mathrm{P}<0.01$.

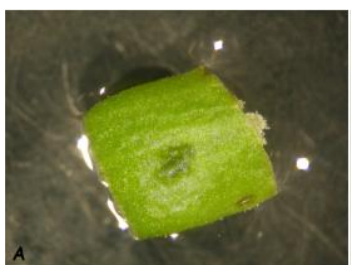

(A)

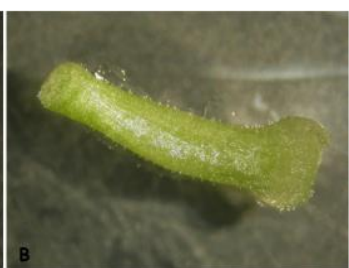

(B)
Figure 1. Explant types used in regeneration, A) Cotyledon explant, B) Hypocotyl explant

\section{Results}

After four weeks of culture, hypocotyl and cotyledon explants of the four $C$. annuum L. lines showed proliferation of multiple shoot formation in indirectly shoot induction media consisting of MS medium supplemented with different concentrations of BAP in combination with IAA (Figure 2).

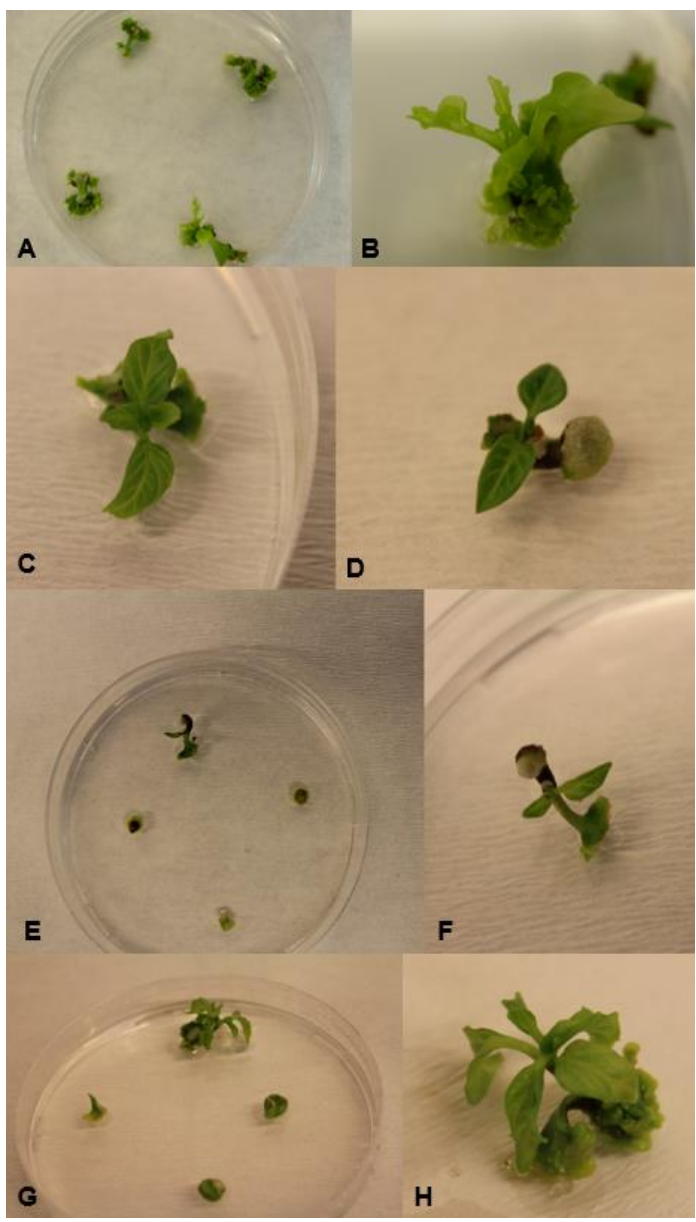

Figure 2. Shoot formation derived from hypocotyl and cotyledon explants

A, B) Formation of shoots from hypocotyl explants after 15 days in 'Green pepper-F614' (MS+4 mg L-1 BAP+0.5 $\mathrm{mg} \mathrm{L}^{-1}$ IAA), C, D) Shoot derived from hypocotyl explant on $\mathrm{MS}+5 \mathrm{mg} \mathrm{L}^{-1} \mathrm{BAP}+0.5 \mathrm{mg} \mathrm{L}^{-1} \mathrm{IAA}$ after 15 days and cotyledon on $2 \mathrm{mg} \mathrm{L}^{-1} \mathrm{BA}+1 \mathrm{mg} \mathrm{L}^{-1}$ IAA after 20 days in 'Capia pepper-M801', E, F) Shoot derived from hypocotyl explant on $\mathrm{MS}+3 \mathrm{mg} \mathrm{L}^{-1} \mathrm{BAP}+0.5 \mathrm{mg} \mathrm{L}^{-1}$ IAA after 15 days in Charleston pepper-

F22' G, H) Shoot derived from hypocotyl explant on MS+5 $\mathrm{mg} \mathrm{L}^{-1}$ BAP+0.5 mg L'-1 IAA after 20 days in 'Bell pepper-F46' 
Table 1. The effect of PGRs and explants on shoot formation in green pepper line

\begin{tabular}{|c|c|c|c|}
\hline \multirow{2}{*}{ PGRs (BA+IAA) mg L ${ }^{-1}$} & \multicolumn{2}{|c|}{ Explants } & \multirow{2}{*}{ PGRs Mean } \\
\hline & Hypocotyl & Cotyledon & \\
\hline $0+0$ (Control) & $0.00^{\mathrm{h}}(0.00)$ & $0.00^{\mathrm{h}}(0.00)$ & $0.00^{\mathrm{G}}(0.00)$ \\
\hline $1+0.5$ & $4.00^{\mathrm{gh}}(5.31)$ & $4.00^{\text {gh }}(5.31)$ & $4.00^{\mathrm{FG}}(5.31)$ \\
\hline $1+1$ & $4.00^{\text {gh }}(5.31)$ & $4.00^{\text {gh }}(5.31)$ & $4.00^{\mathrm{FG}}(5.31)$ \\
\hline $2+0.5$ & $20.00^{\mathrm{def}}(21.00)$ & $16.00^{\mathrm{def}}(21.25)$ & $18.00^{\mathrm{CDE}}(21.12)$ \\
\hline $2+1$ & $12.00^{\mathrm{efg}}(15.93)$ & $32.00^{\text {bcd }}(34.16)$ & $22.00^{\mathrm{BCD}}(25.05)$ \\
\hline $3+0.5$ & $16.00^{\mathrm{efg}}(18.47)$ & $48.00^{\mathrm{b}}(43.84)$ & $32.00^{\mathrm{ABC}}(31.15)$ \\
\hline $3+1$ & $24.00^{\text {cde }}(26.31)$ & $40.00^{\mathrm{bc}}(39.23)$ & $32.00^{\mathrm{AB}}(32.77)$ \\
\hline $4+0.5$ & $80.00^{\mathrm{a}}(66.21)$ & $12.00^{\text {efg }}(15.93)$ & $46.00^{\mathrm{A}}(41.07)$ \\
\hline $4+1$ & $52.00^{\mathrm{b}}(46.15)$ & $8.00^{\text {fgh }}(10.62)$ & $30.00^{\mathrm{BCD}}(28.39)$ \\
\hline $5+0.5$ & $36.00^{\mathrm{bc}}(36.69)$ & $4.00^{\circ} \mathrm{h}(5.31)$ & $20.00^{\mathrm{DE}}(21.00)$ \\
\hline $5+1$ & $24.00^{\text {cde }}(29.09)$ & $0.00^{\mathrm{h}}(0.00)$ & $12.00^{\mathrm{EF}}(14.54)$ \\
\hline Explant Mean & $24.72 \mathrm{~A}(24.59)$ & $6.45)$ & \\
\hline
\end{tabular}

LSD ${ }_{\text {PGR }}: 10.10 * * *$ LSD $_{\text {Explant }}: 4.30 * * *$ LSD $_{\text {PGR Explant }}: 14.28 * * *$, The numbers in parenthesis represent the arcsine transformed value of percentages Differences between means are indicated by the same letters, Control: PGRs-free MS medium, $* \mathrm{P}<0.05, * * \mathrm{P}<0.01, * * * \mathrm{P}<0.001$

Table 2. The effect of PGRs and explants on shoot formation in charleston pepper line

\begin{tabular}{|c|c|c|c|}
\hline \multirow{2}{*}{ PGRs (BA+IAA) $\mathrm{mg} \mathrm{L}^{-1}$} & \multicolumn{2}{|c|}{ Explants } & \multirow{2}{*}{ PGRs Mean } \\
\hline & Hypocotyl & Cotyledon & \\
\hline $0+0$ (Control) & $0.00(0.00)$ & $0.00(0.00)$ & $0.00^{\mathrm{F}}(0.00)$ \\
\hline $1+0.5$ & $0.00(0.00)$ & $4.00^{\mathrm{ij}}(5.31)$ & $2.00^{\mathrm{EF}}(2.65)$ \\
\hline $1+1$ & $0.00(0.00)$ & $12.00^{\mathrm{hi}}(15.93)$ & $6.00^{\mathrm{E}}(7.96)$ \\
\hline $2+0.5$ & $24.00^{\mathrm{fg}}(29.09)$ & $16.00^{\text {gh }}(18.47)$ & $20.00^{\mathrm{CD}}(23.78)$ \\
\hline $2+1$ & $32.00^{\mathrm{ef}}(34.16)$ & $68.00^{\mathrm{ab}}(55.83)$ & $50.00^{\mathrm{B}}(45.00)$ \\
\hline $3+0.5$ & $80.00^{\mathrm{a}}(66.21)$ & $52.00^{\text {bcd }}(46.15)$ & $66.00^{\mathrm{A}}(56.18)$ \\
\hline $3+1$ & $68.00^{\mathrm{ab}}(55.83)$ & $36.00^{\operatorname{def}}(36.69)$ & $52.00^{\mathrm{B}}(46.26)$ \\
\hline $4+0.5$ & $56.00^{\mathrm{bc}}(48.46)$ & $28.00^{\mathrm{ef}}(31.63)$ & $42.00^{\mathrm{B}}(40.04)$ \\
\hline $4+1$ & $52.00^{\text {bcd }}(46.15)$ & $12.00^{\mathrm{hi}}(15.93)$ & $32.00^{\mathrm{C}}(31.04)$ \\
\hline $5+0.5$ & $44.00^{\text {cde }}(41.53)$ & $4.00^{\mathrm{ij}}(5.31)$ & $24.00^{\mathrm{CD}}(23.42)$ \\
\hline $5+1$ & $40.00^{\text {cdef }}(39.23)$ & $4.00^{\mathrm{ij}}(5.31)$ & $22.00^{\mathrm{C}}(22.27)$ \\
\hline Explant Mean & $36.00(32.79)$ & $21.45^{\mathrm{B}}(21.51)$ & \\
\hline
\end{tabular}

Table 3. The effect of PGRs and explants on shoot formation in capia pepper line

\begin{tabular}{|c|c|c|c|}
\hline \multirow{2}{*}{ PGRs (BA+IAA) mg L ${ }^{-1}$} & \multicolumn{2}{|c|}{ Explants } & \multirow{2}{*}{ PGRs Mean } \\
\hline & Hypocotyl & Cotyledon & \\
\hline $0+0$ (Control) & $0.00^{j}(0.00)$ & $0.00^{\mathrm{j}}(0.00)$ & $0.00^{\mathrm{E}}(0.00)$ \\
\hline $1+0.5$ & $16.00^{\text {fgh }}(21.25)$ & $8.00^{\text {hij }}(10.62)$ & $12.00^{\mathrm{CD}}(15.93)$ \\
\hline $1+1$ & $8.00^{\text {hij }}(10.62)$ & $4.00^{\mathrm{ij}}(5.31)$ & $6.00^{\mathrm{DE}}(7.96)$ \\
\hline $2+0.5$ & $28.00^{\text {cdef }}(31.63)$ & $48.00^{\mathrm{bc}}(43.84)$ & $38.00^{\mathrm{A}}(37.73)$ \\
\hline $2+1$ & $24.00^{\text {defg }}(29.09)$ & $36.00^{\text {bcde }}(36.69)$ & $30.00^{\mathrm{AB}}(32.89)$ \\
\hline $3+0.5$ & $32.00^{\text {cdef }}(34.16)$ & $20.00^{\text {efgh }}(23.78)$ & $26.00^{\mathrm{AB}}(28.97)$ \\
\hline $3+1$ & $8.00^{\text {hij }}(10.62)$ & $40.00^{\text {bcd }}(39.00)$ & $24.00^{\mathrm{BC}}(24.81)$ \\
\hline $4+0.5$ & $12.00^{\text {ghi }}(15.93)$ & $16.00^{\text {fgh }}(21.25)$ & $14.00^{\mathrm{C}}(18.59)$ \\
\hline $4+1$ & $36.00^{\text {bcde }}(36.47)$ & $8.00^{\text {hij }}(10.62)$ & $22.00^{\mathrm{BC}}(23.54)$ \\
\hline $5+0.5$ & $80.00^{\mathrm{a}}(66.21)$ & $4.00^{\mathrm{ij}}(5.31)$ & $42.00^{\mathrm{A}}(35.76)$ \\
\hline $5+1$ & $56.00^{\mathrm{b}}(48.46)$ & $0.00^{\mathrm{j}}(0.00)$ & $28.00^{\mathrm{BC}}(24.23)$ \\
\hline Explant Mean & $27.27^{\mathrm{A}}(27.68)$ & $16.72^{\mathrm{B}}(17.86)$ & \\
\hline
\end{tabular}

LSD PGRs $_{9.36 * *}$, LSD Explant: $3.99 * * *$, LSD $_{\text {PGRs*Explant: }}$ 13.23***, The numbers in parenthesis represent the arcsine transformed value of percentages. Differences between means are indicated by the same letters, Control: PGRs-free MS medium, $* \mathrm{P}<0.05, * * \mathrm{P}<0.01, * * * \mathrm{P}<0.001$

The maximum percentage of shoot formation from Bell pepper was obtained in MS medium supplemented with $5 \mathrm{mg}$ $\mathrm{L}^{-1} \mathrm{BA}+0.5 \mathrm{mg} \mathrm{L}^{-1}$ IAA as \%84 (Table 4, Figure 2G, H). For other Capsicum lines of different pepper types, the highest shoot formation was determined as $80 \%$ from hypocotyl explant of green pepper in $4 \mathrm{mg} \mathrm{L}^{-1} \mathrm{BA}+0.5 \mathrm{mg} \mathrm{L}^{-1} \mathrm{IAA}$ (Table 1, Figure 2A, B), 80\% from hypocotyl explant of Charleston pepper in $3 \mathrm{mg} \mathrm{L}^{-1} \mathrm{BA}+0.5 \mathrm{mg} \mathrm{L}^{-1} \mathrm{IAA}$ (Table 2, Figure 2E, F) and $80 \%$ from hypocotyl explant of capia pepper in $5 \mathrm{mg} \mathrm{L}^{-1} \mathrm{BA}+0.5 \mathrm{mg} \mathrm{L}^{-1}$ (Table 3, Figure 2D).
Shoot formation obtained from different explants of Capsicum lines on MS media containing different concentrations and combinations of BAP and IAA did not elongate of shoots development when continued to culture on the same medium. Frequently, shoot structures were transferred to shoot elongation medium in vitro because shoot elongation has been found as major obstacle in obtaining normal pepper plant (Steinitz et al., 1999). Cytokinins generally stimulate shoot proliferation and inhibit their elongation. The problem of shoot elongation might be due to transferring of shoot structures 
on different media (Peddaboina et al., 2003). In this study, different concentrations of BAP and IAA were used. $\mathrm{GA}_{3}(2 \mathrm{mg}$ $\mathrm{L}^{-1}$ ) was used to stimulate shoot elongation in the media. Therefore, the MS medium supplemented with $2.0 \mathrm{mg} \mathrm{L}^{-1} \mathrm{GA}_{3}$ showed the highest effect on shoot elongation when compared to BAP and IAA combination or PGR-free medium.

Among the PGR combinations, callus formation of hypocotyl and cotyledon was the lowest in BAP and IAA combination. However, between the two explants, cotyledon had higher potentiality of callus induction than hypocotyl in all Capsicum lines of different types. However, cotyledon explants showed higher callus formation than hypocotyl. No shoot was observed from calli coming from cotyledon explants. And calli formation did not show any regeneration capability. Callus formation ratio was as follows; in green pepper type-from cotyledon explant-with $1 \mathrm{mg} \mathrm{L}^{-1} \mathrm{BAP}+1 \mathrm{mg} \mathrm{L}^{-1} \mathrm{IAA}$, as $28 \%$ (Table $5)$, in Charleston pepper- type cotyledon explant-with $1 \mathrm{mg}$ $\mathrm{L}^{-1} \mathrm{BAP}+0.5 \mathrm{mg} \mathrm{L}^{-1}$ IAA as $16 \%$ (Table 6), capia pepper type- cotyledon explant- with $1 \mathrm{mg} \mathrm{L}^{-1} \mathrm{BAP}+0.5 \mathrm{mg} \mathrm{L}^{-1}$ IAA as $28 \%$ (Table 7), bell pepper type-cotyledon explant$1 \mathrm{mg} \mathrm{L}^{-1} \mathrm{BAP}+0.5 \mathrm{mg} \mathrm{L}^{-1}$ IAA as $36 \%$ (Table 8). The size of calli was smaller, friable and yellowish on these media for all Capsicum lines of different types.

Table 4. The effect of PGRs and explants on shoot formation in bell pepper line

\begin{tabular}{l|lcc}
\hline \multirow{2}{*}{ PGRs (BA+IAA) $\mathrm{mg} \mathrm{L}^{-1}$} & \multicolumn{2}{c}{ Explants } & \multirow{2}{*}{ PGRs Mean } \\
\cline { 2 - 3 } & Hypocotyl & $0.00^{\mathrm{f}}(0.00)$ & $0.00^{\mathrm{C}}(0.00)$ \\
$1+0.5$ & $0.00^{\mathrm{f}}(0.00)$ & $4.00^{\mathrm{f}}(5.31)$ & $4.00^{\mathrm{C}}(5.31)$ \\
$1+1$ & $4.00^{\mathrm{f}}(5.31)$ & $8.00^{\mathrm{f}}(10.62)$ & $4.00^{\mathrm{C}}(5.31)$ \\
$2+0.5$ & $0.00^{\mathrm{f}}(0.00)$ & $32.00^{\mathrm{cde}}(34.16)$ & $32.00^{\mathrm{A}}(34.16)$ \\
$2+1$ & $32.00^{\text {cde }}(34.16)$ & $52.00^{\mathrm{b}}(46.15)$ & $38.00^{\mathrm{A}}(37.62)$ \\
$3+0.5$ & $24.00^{\mathrm{de}}(29.09)$ & $44.00^{\mathrm{bc}}(41.53)$ & $38.00^{\mathrm{A}}(37.85)$ \\
$3+1$ & $32.00^{\text {cde }}(34.16)$ & $40.00^{\mathrm{bcd}}(39.00)$ & $42.00^{\mathrm{A}}(40.27)$ \\
$4+0.5$ & $44.00^{\mathrm{bc}}(41.53)$ & $20.00^{\mathrm{e}}(26.56)$ & $36.00^{\mathrm{A}}(34.96)$ \\
$4+1$ & $52.00^{\mathrm{b}}(46.15)$ & $20.00^{\mathrm{e}}(26.56)$ & $38.00^{\mathrm{A}}(37.51)$ \\
$5+0.5$ & $56.00^{\mathrm{b}}(48.46)$ & $8.00^{\mathrm{f}}(10.62)$ & $46.00^{\mathrm{A}}(39.68)$ \\
$5+1$ & $84.00^{\mathrm{a}}(68.74)$ & $4.00^{\mathrm{f}}(5.31)$ & $16.00^{\mathrm{B}}(18.47)$ \\
\hline Explant Mean & $28.00^{\text {cde }}(31.63)$ & $21.09^{\mathrm{B}}(22.09)$ & \\
\hline
\end{tabular}

LSD $_{\text {Explant }}: 3.28 * * *$, LSD $_{\text {PGRs }}: 7.70^{* * *}$, LSD Explant*PGs: $_{10.89 * * *}$, The numbers in parenthesis represent the arcsine transformed value of percentages. Differences between means are indicated by the same letters, Control: PGRs-free MS medium, $* \mathrm{P}<0.05, * * \mathrm{P}<0.01, * * * \mathrm{P}<0.001$

Table 5. The effect of PGRs and explants on callus formation in green pepper line

\begin{tabular}{l|ccc}
\hline \multirow{2}{*}{ PGRs (BA+IAA) $\mathrm{mg} \mathrm{L}^{-1}$} & \multicolumn{2}{c}{ Explants } & \multirow{2}{*}{ PGRs Mean } \\
\cline { 2 - 3 } & Hypocotyl & $0.00^{\mathrm{c}}(0.00)$ & $0.00^{\mathrm{C}}(0.00)$ \\
$1+0$ (Control) & $0.00^{\mathrm{c}}(0.00)$ & $12.00^{\mathrm{b}}(15.93)$ & $8.00^{\mathrm{B}}(10.62)$ \\
$1+1$ & $4.00^{\mathrm{c}}(5.31)$ & $28.00^{\mathrm{a}}(28.85)$ & $24.00^{\mathrm{A}}(27.70)$ \\
$2+0.5$ & $20.00^{\mathrm{a}}(26.56)$ & $0.00^{\mathrm{c}}(0.00)$ & $0.00^{\mathrm{C}}(0.00)$ \\
$2+1$ & $0.00^{\mathrm{c}}(0.00)$ & $0.00^{\mathrm{c}}(0.00)$ & $0.00^{\mathrm{C}}(0.00)$ \\
$3+0.5$ & $0.00^{\mathrm{c}}(0.00)$ & $0.00^{\mathrm{c}}(0.00)$ & $0.00^{\mathrm{C}}(0.00)$ \\
$3+1$ & $0.00^{\mathrm{c}}(0.00)$ & $0.00^{\mathrm{c}}(0.00)$ & $0.00^{\mathrm{C}}(0.00)$ \\
$4+0.5$ & $0.00^{\mathrm{c}}(0.00)$ & $0.00^{\mathrm{c}}(0.00)$ & $0.00^{\mathrm{C}}(0.00)$ \\
$4+1$ & $0.00^{\mathrm{c}}(0.00)$ & $0.00^{\mathrm{c}}(0.00)$ & $0.00^{\mathrm{C}}(0.00)$ \\
$5+0.5$ & $0.00^{\mathrm{c}}(0.00)$ & $0.00^{\mathrm{c}}(0.00)$ & $0.00^{\mathrm{C}}(0.00)$ \\
$5+1$ & $0.00^{\mathrm{c}}(0.00)$ & $0.00^{\mathrm{c}}(0.00)$ & $0.00^{\mathrm{C}}(0.00)$ \\
\hline Explant Mean & $0.00^{\mathrm{c}}(0.00)$ & $3.63^{\mathrm{A}}(4.07)$ & \\
\hline
\end{tabular}

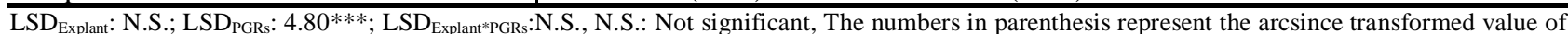
percentages. Differences between means are indicated by the same letters, Control: PGRs-free MS medium, $* \mathrm{P}<0.05, * * \mathrm{P}<0.01, * * * \mathrm{P}<0.001$

Table 6. The effect of PGRs and explants on callus formation in charleston pepper line

\begin{tabular}{l|lcl}
\hline \multirow{2}{*}{ PGRs (BA+IAA) mg L } & \multicolumn{2}{c}{ Explants } & \multirow{2}{*}{ PGRs Mean } \\
\cline { 2 - 3 } & Hypocotyl & Cotyledon & $0.00^{\mathrm{B}}(0.00)$ \\
$1+0.5$ & $0.00(0.00)$ & $0.00(0.00)$ & $14,00^{\mathrm{A}}(18.59)$ \\
$1+1$ & $12.00^{\mathrm{ab}}(15.93)$ & $16.00^{\mathrm{a}}(21.25)$ & $10,00^{\mathrm{A}}(13.28)$ \\
$2+0.5$ & $8.00^{\mathrm{bc}}(10.62)$ & $12.00^{\mathrm{ab}}(15.93)$ & $0.00^{\mathrm{B}}(0.00)$ \\
$2+1$ & $0.00^{\mathrm{d}}(0.00)$ & $0.00^{\mathrm{d}}(0.00)$ & $2,00^{\mathrm{B}}(2.65)$ \\
$3+0.5$ & $4.00^{\mathrm{d}}(5.31)$ & $0.00^{\mathrm{d}}(0.00)$ & $0.00^{\mathrm{B}}(0.00)$ \\
$3+1$ & $0.00^{\mathrm{d}}(0.00)$ & $0.00^{\mathrm{d}}(0.00)$ & $0.00^{\mathrm{B}}(0.00)$ \\
$4+0.5$ & $0.00^{\mathrm{d}}(0.00)$ & $0.00^{\mathrm{d}}(0.00)$ & $0.00^{\mathrm{B}}(0.00)$ \\
$4+1$ & $0.00^{\mathrm{d}}(0.00)$ & $0.00^{\mathrm{d}}(0.00)$ & $0.00^{\mathrm{B}}(0.00)$ \\
$5+0.5$ & $0.00^{\mathrm{d}}(0.00)$ & $0.00^{\mathrm{d}}(0.00)$ & $0.00^{\mathrm{B}}(0.00)$ \\
$5+1$ & $0.00^{\mathrm{d}}(0.00)$ & $0.00^{\mathrm{d}}(0.00)$ & $0.00^{\mathrm{B}}(0.00)$ \\
\hline Explant Mean & $0.00^{\mathrm{d}}(0.00)$ & $0.00^{\mathrm{d}}(0.00)$ & $2.54^{\mathrm{A}}(3.38)$ \\
\hline
\end{tabular}

LSD $_{\text {Eksplant }}$ N.S.; LSD PGRs $_{1}: 5.73 * * * ;$ LSD $_{\text {Explant*PGs: }}$ N.S., N.S.: Not significant, The numbers in parenthesis represent the arcsine transformed value of percentages. Differences between means are indicated by the same letters, Control: PGRs-free MS medium, $* \mathrm{P}<0.05, * * \mathrm{P}<0.01, * * * \mathrm{P}<0.001$ 
Table 7. The effect of PGRs and explants on callus formation in capia pepper line

\begin{tabular}{l|ccc}
\hline \multirow{2}{*}{ PGRs (BA+IAA) $\mathrm{mg} \mathrm{L}^{-1}$} & \multicolumn{2}{c}{ Explants } & \multirow{2}{*}{ PGRs Mean } \\
\cline { 2 - 3 } $0+0$ (Control) & $0.00^{\mathrm{d}}(0.00)$ & $0.00^{\mathrm{d}}(0.00)$ & $0.00^{\mathrm{C}}(0.00)$ \\
$1+0.5$ & $20.00^{\mathrm{ab}}(26.56)$ & $28.00^{\mathrm{a}}(31.63)$ & $24.00^{\mathrm{A}}(29.09)$ \\
$1+1$ & $12.00^{\mathrm{c}}(15.93)$ & $20.00^{\mathrm{b}}(23.78)$ & $16.00^{\mathrm{B}}(19.86)$ \\
$2+0.5$ & $0.00^{\mathrm{d}}(0.00)$ & $0.00^{\mathrm{d}}(0.00)$ & $0.00^{\mathrm{C}}(0.00)$ \\
$2+1$ & $0.00^{\mathrm{d}}(0.00)$ & $0.00^{\mathrm{d}}(0.00)$ & $0.00^{\mathrm{C}}(0.00)$ \\
$3+0.5$ & $0.00^{\mathrm{d}}(0.00)$ & $0.00^{\mathrm{d}}(0.00)$ & $0.00^{\mathrm{C}}(0.00)$ \\
$3+1$ & $0.00^{\mathrm{d}}(0.00)$ & $0.00^{\mathrm{d}}(0.00)$ & $0.00^{\mathrm{C}}(0.00)$ \\
$4+0.5$ & $0.00^{\mathrm{d}}(0.00)$ & $0.00^{\mathrm{d}}(0.00)$ & $0.00^{\mathrm{C}}(0.00)$ \\
$4+1$ & $0.00^{\mathrm{d}}(0.00)$ & $0.00^{\mathrm{d}}(0.00)$ & $0.00^{\mathrm{C}}(0.00)$ \\
$5+0.5$ & $0.00^{\mathrm{d}}(0.00)$ & $0.00^{\mathrm{d}}(0.00)$ & $0.00^{\mathrm{C}}(0.00)$ \\
$5+1$ & $0.00^{\mathrm{d}}(0.00)$ & $0.00^{\mathrm{d}}(0.00)$ & $0.00^{\mathrm{C}}(0.00)$ \\
Explant Mean & $2.90^{\mathrm{A}}(3.86)$ & $4.36^{\mathrm{A}}(5.03)$ & \\
\hline
\end{tabular}

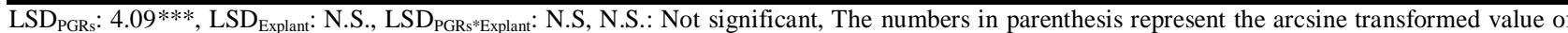
percentages. Differences between means are indicated by the same letters, Control: PGRs-free MS medium, $* \mathrm{P}<0.05, * * \mathrm{P}<0.01, * * * \mathrm{P}<0.001$

Table 8. The effect of PGRs and explants on callus formation in bell pepper line

\begin{tabular}{l|ccc}
\hline \multirow{2}{*}{ PGRs (BA+IAA) $\mathrm{mg} \mathrm{L}^{-1}$} & \multicolumn{2}{c}{ Explants } & \multirow{2}{*}{ PGRs Mean } \\
\cline { 2 - 3 } & Hypocotyl & Cotyledon & $0.00^{\mathrm{C}}(0.00)$ \\
\hline$+0($ Control) & $0.00^{\mathrm{d}}(0.00)$ & $0.00^{\mathrm{d}}(0.00)$ & $26.00^{\mathrm{A}}(27.58)$ \\
$1+0.5$ & $16.00^{\mathrm{c}}(18.47)$ & $36.00^{\mathrm{a}}(36.69)$ & $18.00^{\mathrm{B}}(22.51)$ \\
$2+0.5$ & $12.00^{\mathrm{c}}(15.93)$ & $24.00^{\mathrm{b}}(29.09)$ & $0.00^{\mathrm{C}}(0.00)$ \\
$2+1$ & $0.00^{\mathrm{d}}(0.00)$ & $0.00^{\mathrm{d}}(0.00)$ & $0.00^{\mathrm{C}}(0.00)$ \\
$3+0.5$ & $0.00^{\mathrm{d}}(0.00)$ & $0.00^{\mathrm{d}}(0.00)$ & $0.00^{\mathrm{C}}(0.00)$ \\
$3+1$ & $0.00^{\mathrm{d}}(0.00)$ & $0.00^{\mathrm{d}}(0.00)$ & $0.00^{\mathrm{C}}(0.00)$ \\
$4+0.5$ & $0.00^{\mathrm{d}}(0.00)$ & $0.00^{\mathrm{d}}(0.00)$ & $0.00^{\mathrm{C}}(0.00)$ \\
$4+1$ & $0.00^{\mathrm{d}}(0.00)$ & $0.00^{\mathrm{d}}(0.00)$ & $0.00^{\mathrm{C}}(0.00)$ \\
$5+0.5$ & $0.00^{\mathrm{d}}(0.00)$ & $0.00^{\mathrm{d}}(0.00)$ & $0.00^{\mathrm{C}}(0.00)$ \\
$5+1$ & $0.00^{\mathrm{d}}(0.00)$ & $0.00^{\mathrm{d}}(0.00)$ & $0.00^{\mathrm{C}}(0.00)$ \\
Explant Mean & $0.00^{\mathrm{d}}(0.00)$ & $0.00^{\mathrm{d}}(0.00)$ & \\
\hline
\end{tabular}

LSD Explant: $1.95 * *$, LSD $_{\text {PGRs }}: 4.59 * * *$, LSD Explant*PGs $: 6.49 * * *$, The numbers in parenthesis represent the arcsine transformed value of percentages. Differences between means are indicated by the same letters, Control: PGRs-free MS medium, $* \mathrm{P}<0.05, * * \mathrm{P}<0.01, * * * \mathrm{P}<0.001$

The shoots multiplicated showed rooting and elongation in MS medium supplemented with $0.5 \mathrm{mg} \mathrm{L}^{-1}$ IAA. These plantlets rooted were cut again and used for further micropropagation (Figure 3). Rapid proliferated of the plantlets was achieved within a short span of time.

\section{Discussion}

The shoot structures were generally obtained from nutrient media containing high cytokinin (3-5 mg L-1 $\mathrm{BA})$ and low auxin $\left(0.5 \mathrm{mg} \mathrm{L}^{-1}\right.$ IAA). When the literature is examined, BA and IAA are the most commonly used plant growth regulators and combinations in organogenesis studies in Capsicum (Gunay and Rao, 1978; Agrawal et al., 1989; Arroyo and Revilla, 1991; Christopher and Rajam, 1996; Ramírez-Malagón and Ochoa-Alejo, 1996; Hyde and Phillips, 1996; Sanatombi and Sharma, 2006; Sanatombi and Sharma, 2008; Renfiyeni et al., 2017; Haque and Ghosh, 2018; Gammoudi et al., 2017). We observed that the shoot structures were generally obtained from nutrient media containing high cytokinin $\left(3-5 \mathrm{mg} \mathrm{L}^{-1}\right.$ BA) and low auxin (0.5 $\mathrm{mg} \mathrm{L}^{-1}$ IAA). Gunay and Rao (1978) conducted the first organogenesis studies in Capsicum. They also obtained the best shoot growth in cotyledon explants using $2 \mathrm{mg} \mathrm{L}^{-1} \mathrm{BA}+1 \mathrm{mg} \mathrm{L}^{-1}$ IAA such as this research presented. Researchers have reported that the combination of BA and IAA is effective on shoot formation. Similar results were obtained in this study. In particular, the combination of BA and IAA promoted shoot formation effectively in four pepper lines of different types and hypocotyl explants were found to respond better than cotyledon explants in shoot formation. In organogenesis studies performed on eight different pepper genotypes $(C$. praeternissum, $C$. baccatum and $C$. annuum cvs. G4, Bhiwapuri, Sweet pepper, Cayenne pepper and hybrid pepper), leaf explants were successful (56\%) than cotyledon and hypocotyl explants (Christopher and Rajam, 1996). Researchers have reported that the use of BA alone is more effective than the combination of BA and IAA for shoot formation (Christopher and Rajam, 1996). In this study, the use of BA alone was found to be insufficient in shoot formation in four pepper lines of different types. In regeneration experiments, MS medium without PGRs was used in all control groups. However, any improvement was not observed in hypocotyl and cotyledon explants. In contrast to this, Ramírez-Malagón and Ochoa-Alejo (1996) obtained the best shoot growth in Chilean pepper from MS medium without PGRs (26.8/per explant). This situation shows differences from our research results. The results of these researchers are not consistent with both our study and the recent studies on organogenesis. Regarding Capsicum genotypes, it is reported that regeneration is still difficult and genotype effect plays an important role. In this research presented, the highest shoot formation (average 
$80 \%)$ was obtained in BA (3-5 $\left.\mathrm{mg} \mathrm{L}^{-1}\right)$ and IAA $\left(0.5 \mathrm{mg} \mathrm{L}^{-1}\right)$ combinations in four pepper lines of different types. Ashwani et al. (2017) reported that shoot formation was obtained from the medium with $10 \mathrm{mg} \mathrm{L}^{-1} \mathrm{BA}+1 \mathrm{mg} \mathrm{L}^{-1}$ IAA using proximal parts of the cotyledon leaves as $60 \%$, while petiole explants showed $40 \%$ shoot formation ratio. In the proximal parts of the cotyledon leaves, $5 \mathrm{mg} \mathrm{L}^{-1} \mathrm{BA}+2 \mathrm{mg} \mathrm{L}^{-1}$ phenyl acetic acid (PAA) attained $100 \%$ shoot formation. Ashwani et al. (2017) achieved an effective result by reducing $\mathrm{BA}$ concentration from $10 \mathrm{mg} \mathrm{L}^{-1}$ to $5 \mathrm{mg} \mathrm{L}^{-1}$ and using PAA as a different auxin. However, PAA seems to be more effective than IAA in this medium. This may be due to the different Capsicum species used. Several studies have reported that IAA is an effective auxin for organogenesis studies in C. annuum L. However, the genotype effect is still an important factor in Capsicum genus (Venkataiah and Subhash, 2001; Renfiyeni et al., 2017).

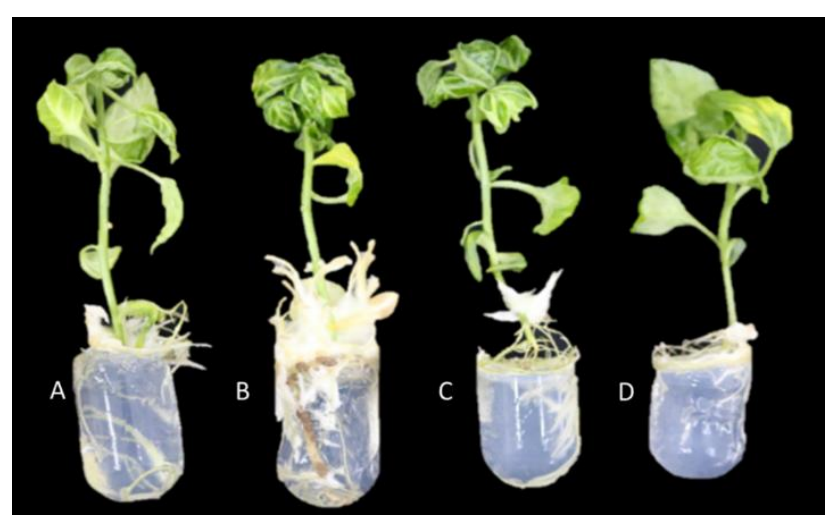

Figure 3. In vitro regeneration and induction of rooted plantlets in Capsicum annuum $\mathrm{L}$.

A) 'Green pepper-F614', B) 'Charleston pepper-F22', C) 'Capia pepperM801', D) 'Bell pepper-F46'

\section{Conclusion}

In this study, for direct organogenesis, hypocotyl and cotyledon explants from 11-day-old seedlings and MS medium supplemented with $3-5 \mathrm{mg} \mathrm{L}^{-1} \mathrm{BAP}+0.5 \mathrm{mg} \mathrm{L}^{-1}$ IAA was found to be successful for all Capsicum lines of different pepper types. The present research was successful in obtaining shoot initiation and elongation. Plant regeneration through direct organogenesis is a very quick process. Therefore, direct regeneration from hypocotyl explant is very effective and time saver.

\section{Acknowledgments}

The author (T.I.) is grateful to the Graduate School of Natural and Applied Science of Ege University (İzmir, Turkey) to support his $\mathrm{PhD}$ thesis.

\section{References}

Aboshama HMS. 2011. Direct somatic embryogenesis of pepper (Capsicum annuиm L.). World Journal of Agricultural Sciences, 7(6):755-762.

Agrawal S, Chandra N. 1983. Differentiation of multiple shoot buds and plantlets in culture embryos of Capsicum annuum L. var. Mathania. Curr. Sci., 52: 645-46.

Agrawal S, Chandra N, Kothari SL. 1989. Plant regeneration in tissue cultures of pepper (Capsicum annuum L. cv. Mathania). Plant Cell Tiss. Org., 16: 47-55.
Arous S, Boussaid M, Marrakchi M. 2001. Plant regeneration from zygotic embryo hypocotyls of Tunisian chili (Capsicum annuиm L.). Journal of Applied Horticulture, 3: 17-22.

Akyavuz R, Taskin B, Koçak M, Yildiz M. 2018. Exploring the genetic variations and population structure of Turkish pepper (Capsicum annuиm L.) genotypes based on peroxidase gene markers. 3 Biotech, 8(8): 355. doi: 10.1007/s13205-0181380-2

Arroyo R, Revilla MA. 1991. In vitro plant regeneration from cotyledon and hypocotyl segments in two bell pepper cultivars. Plant Cell Rep., 10: 414-416.

Ashwani S, Ravishankar GA, Giridhar P. 2017. Silver nitrate and 2-(N-morpholine) ethane sulphonic acid in culture medium promotes rapid shoot regeneration from the proximal zone of the leaf of Capsicum frutescens Mill. Plant Cell Tiss. Org., 129(1): 175-180.

Bhutia KL, Meetei NT, Khanna VK. 2018. In vitro direct regeneration of Dalle Khursani (Capsicum annum) from salicylic acid treated explants. Journal of Pharmacognosy and Phytochemistry, 7(2): 1008-1012.

Christopher T, Rajam MV. 1994. In vitro clonal propagation of Capsicum spp. Plant Cell Tiss. Org., 38:25-9.

Christopher T, Rajam MV. 1996. Effect of genotype, explant and medium on in vitro regeneration of red pepper. Plant Cell Tiss. Org., 46: 245-50.

Dabauza M, Pena L. 2001. High efficiency organogenesis in sweet pepper (Capsicum annuиm L.) tissues from different seedlings explants. Plant Growth Regul., 33: 221-9.

Ebida AIA, Hu CY. 1993. In vitro morphogenetic responses and plant regeneration from pepper (Capsicum annuum L. cv. Early California Wonder) seedling explant. Plant Cell Rep., 13:107-10.

El Nagar MM. 2012. Somatic embryogenesis of pepper (Capsicum annuum L.) and regeneration of transgenic plants after Agrobacterium-Mediated transformation. Journal of Applied Sciences Research, 8(11): 5550-5563.

Ezura H, Nishimiya S, Kasumi M. 1993. Efficient regeneration of plants independent of exogenous growth regulators in bell pepper (Capsicum annuum L.). Plant Cell Rep., 12: 676-80.

Franck-Duchenne M, Wang Y, Tahar SB, Beachy RN. 1998. In vitro stem elongation of sweet pepper in media containing 24epi-brassinolide. Plant Cell Tiss. Org., 53(2): 79-84.

Gammoudi N, San Pedro T, Ferchichi A, Gisbert C. 2017. Improvement of regeneration in pepper: a recalcitrant species. In Vitro Cell Dev-Pl., 54(2):145-153.

Gunay AL, Rao P. 1978. In vitro plant regeneration from hypocotyl and cotyledon explants of red pepper (Capsicum). Plant Sci. Lett., 11: 365-72.

Haque SKM, Ghosh B. 2018. An improved micropropagation protocol for the recalcitrant plant Capsicum - a study with ten cultivars of Capsicum spp. (C. annuum, C. chinense, and $C$. frutescens) collected from diverse geographical regions of India and Mexico. The Journal of Horticultural Science and Biotechnology, 93: 1:91-99.

Hegde V, Partap PS, Yadav RC. 2017a. In vitro regeneration of Capsicum (Capsicum annuum L.) from cotyledon explants. International Journal of Current Microbiology and Applied Sciences, 6(5): 225-237.

Hegde V, Partap PS, Yadav RC. 2017b. Plant regeneration from hypocotyl explants in capsicum (Capsicum annuum L.). International Journal of Current Microbiology and Applied Sciences, 6(7): 545-557.

Husain S, Jain A, Kothari SL. 1999. Phenylacetic acid improves bud elongation and in vitro plant regeneration efficiency in Capsicum annuum L. Plant Cell Rep., 19: 64-8.

Hyde C, Phillips GC. 1996. Silver nitrate promotes shoot development and plant regeneration on chile pepper (Capsicum annuum L.) via organogenesis. In Vitro Cell DevPl.,32: 72-80. 
Joshi A, Kothari SL. 2007. Copper levels in the medium improves differentiation and elongation from cultured cotyledons of Capsicum annuum L. Plant Cell Tiss. Org., 88: 127-33.

Kothari SL, Joshi A, Kachhwaha S, Ochoa-Alejo N. 2010. Chilli peppers - a review on tissue culture and transgenesis. Biotechnol. Adv., 28(1): 35-48.

Liu W, Parrott WA, Hildebrand DF, Collins GB, Williams EG. 1990. Agrobacterium induced gall formation in bell pepper (Capsicum annuиm L.) and formation of shoot-like structures expressing introduced genes. Plant Cell Rep., 9: 360-4.

Madhuri V, Rajam MV. 1993. Apical shoot meristemculture in red pepper (Capsicum annuum L.). J. Plant Biochem. Biot., 2:67-8.

Maligeppagol M, Manjula R, Navale PM, Babu KP, Kumbar BM, Laxman RH. 2016. Genetic transformation of chilli (Capsicum annuum L.) with Dreb1A transcription factor known to impart drought tolerance. Indian J. Biotechnol., 15(1): 17-24.

Murashige T, Skoog F. 1962. A resived medium for rapid growth and bioassays with tobacco tissue cultures. Physiol. Plantarum, 84(15): 473-497.

Ochoa-Alejo N, Ramírez-Malagón R. 2001. In vitro chili pepper biotechnology. In Vitro Cell Dev-P1., 37(6): 701-729.

Orlinska M, Nowaczyk P. 2015. In vitro plant regeneration of 4 Capsicum spp. genotypes using different explant types. Turk. J. Biol., 39(1): 60-68.

Peddaboina V, Thamidala C, Karampuri S. 2003. Thidiazuron induced high frequency adventitious shoot formation and plant regeneration in Capsicum annuиm L. Journal of Plant Biotechnology, 5(4):245-250.
Phillips GC, Hubstenberger JF. 1985. Organogenesis in pepper tissue cultures. Plant Cell Tiss. Org., 4: 261-9.

Ramírez-Malagón R, Ochoa-Alejo N. 1996. An improved and reliable chilli pepper (Capsicum annuum L.) plant regeneration method. Plant Cell Rep., 16: 226-31.

Renfiyeni S, Syukriani L, Asben A, Jamsari J. 2017. Optimal media for in-vitro regeneration of two local genotypes of chili pepper (Capsicum annuum L.) from West Sumatera. International Journal on Advanced Science, Engineering and Information Technology, 7(3): 904-909.

Sanatombi K, Sharma GJ. 2006. In vitro regeneration and mass multiplication of Capsicum annuиm L. Journal of Food, Agriculture and Environment, 4: 205-8.

Sanatombi K, Sharma GJ. 2007a. Micropropagation of Capsicum frustescens L. using axillary shoot explants. Sci. Hortic., 113(1): 96-99.

Sanatombi K, Sharma GJ. 2007b. Micropropagation of Capsicum annum L. Not. Bot. Horti Agrobo., 35(1): 255-965.

Sanatombi K, Sharma GJ. 2008. In vitro plant regeneration in six cultivars of Capsicum spp. using different explants. Biol. Plantarum, 52(1):141-145.

Steinitz B, Wolf D, Matzevitch-Josef T, Zelcer A. 1999. Regeneration in vitro and genetic transformation of pepper (Capsicum spp.): The current state of art. Capsicum and Eggplant Newsletter, 18: 9-15.

Valadez-Bustos MG, Aguado-Santacruz GA, Carrillo-Castañeda G, Aguilar-Rincón VH, Espitia-Rangel E, Montes-Hernández S, Robledo-Paz A. 2009. In vitro propagation and agronomic performance of regenerated chili pepper (Capsicum spp.) plants from commercially important genotypes. In Vitro Cell Dev-Pl., 45:650. doi:10.1007/s11627-009-9193-y 